\title{
Soft Components for Soft Robots
}

\author{
Jamie Paik \\ Head of Reconfigurable Robotics Lab, \\ Ecole Polytechnique Federale de Lausanne, Switzerland.
}

\begin{abstract}
Typical robot platforms comprise rigid links with fixed degrees-offreedom, solid blocks of transmission and actuator, and superficial positioning of sensors: they are often optimized for the given design criteria but are unable to execute instantaneous changes to the robot's initial mechanism design. The reallife incidences, however, require robots to face complex situations filled with unprogrammed tasks and unforeseen environmental changes. One of the growing efforts in the field that address such juxtaposing design paradigm is soft robotics: augmentations of "softness" in robots to complement, adapt, and reconfigure to the contingent assignments. Although the "softness" invokes and relates to many facets of robot design in both soft and hardware, this manuscript focuses on describing some critical hardware components. I will present several on going research on actuation and sensor solutions for soft robotics application as well as novel methods and materials for sensor and actuation integration.
\end{abstract}

\section{Introduction: What kind of Softness?}

The general concept of soft robotics stimulates interest from a wide field of scientists for its projection of autonomous systems that can safely conform to the unknown environment and un-programmed tasks. While the word "softness" attracts diverse interpretations, it connotes two aspects: intrinsic and extrinsic softness of the said mechanism. The intrinsic softness signals the compliancy achieved from the material characteristics where the level of the softness highly depends on the Young's modulus (<100MPa) of the main composing material. The extrinsic softness relates to the increased compliancy of the body and/or endeffector through mechanism design (i.e. springs, compliant joints). Evidently, a purely intrinsically soft robot suffers from reduced zero force bandwidth while a thoroughly extrinsic one has limited reconfigurability large or small. Therefore, for a truly interactive and soft system, optimal consolidation of both ends of the "softness" spectrum is crucial, followed by seamless integration of the multiple "soft" components. For any robotic system, the principal design boundaries come from the mechanical performance and capacity of the actuator, sensor and the control solutions. In the following sections, I will focus on actuator and sensor solutions that are feasible for often unconventional soft robot designs. 


\section{Actuators for Soft Robots}

Most conventional actuators maintain the interface with its load as stiff as possible to maximize the work and control efficiency. For soft robots, reducing or even eliminating this interface stiffness introduces safety via lower reflected inertia, more stable force control (although the accuracy could be debatable), less inadvertent damage to the environment, the capacity for energy storage, and a high reconfigurability.

\subsection{Actuators for Multi-DoF Designs}

Mechanisms with multiple compliant joints or augmented degree-of-freedom (DoF) require specific transmissions linked with novel actuators that meet the design restrictions. There have been examples of modular connection of serial kinematic based actuators and / or foldable structures using flexible joints. The serial kinematic based actuators can have fully actuated joint; however, underactuated systems are preferred for reducing the mechanism complexity while enhancing the flexibility. There are several ways of adding transmission or (under) actuating these passive joints: they can either be flexures [1] or springs [2], and pro / antagonistic cables, or encapsulated geartrain that are controlled by a single actuator. Developed in the early 90's and continuing to evolve, series elastic actuators (SEAs) provide variable and controllable compliance using mechanical springs / compliant elements within the serial connection [3-5]. The elongation of the spring is used as the force measurement. When considering transmission-less actuations, smart materials like shape memory alloys (SMA) or magneto-, thermorheological fluids [6] that locally modulate the stiffness of joints are attractive choices. For direct actuation with restrictive body configuration, thermally activated foldable SMA actuators are effective for various types of 2D origami robots [7-11]. Depending on the application, the motion patterns are set while SMA wires [7,8], SMA sheets $[9,10]$, and conductive polymer films [11] actuate the individual joint.

\subsection{Pneumatic Artificial Muscles (PAMs)}

PAMs, also known as Mckibben actuators, are basically pressurized air-filled rubber bladders with valves that control overall range of motion and applied force / torque: the compliance of the actuator can vary via operating pressure. The most commonly used PAMs are cylindrical balloons with rigid metal valves and shaft attachments to ensure the volume to linear displacement transmission [12, 13]. In order to increase the contraction force, PAMs with embedded fibers in the rubber 
air chamber are also introduced [14-16]. While the physics of the actuation principle is similar, soft pneumatic actuators (SPAs)' uniqueness comes from their air chambers that are entirely made of much softer silicone $\left(\mathrm{PDMS}^{\mathrm{TM}}, \mathrm{Ecoflex}^{\mathrm{TM}}\right.$ ) instead of rubber (about 1/100 of Young's modulus); therefore, the operating pressure is a fraction of atmospheric pressure making them more compliant and safer. Owing to the new material and construction, these recent generation of pressure driven actuators are also highly customizable: their sizes and functionality (range of motion, compliance, torque / force output, actuation points) can be engineered by the geometry of the mold while the intrinsic softness can still be addressed via switching the silicone hardness [17, 18]. SPAs are effective even with fluids instead of pressurized air [19].

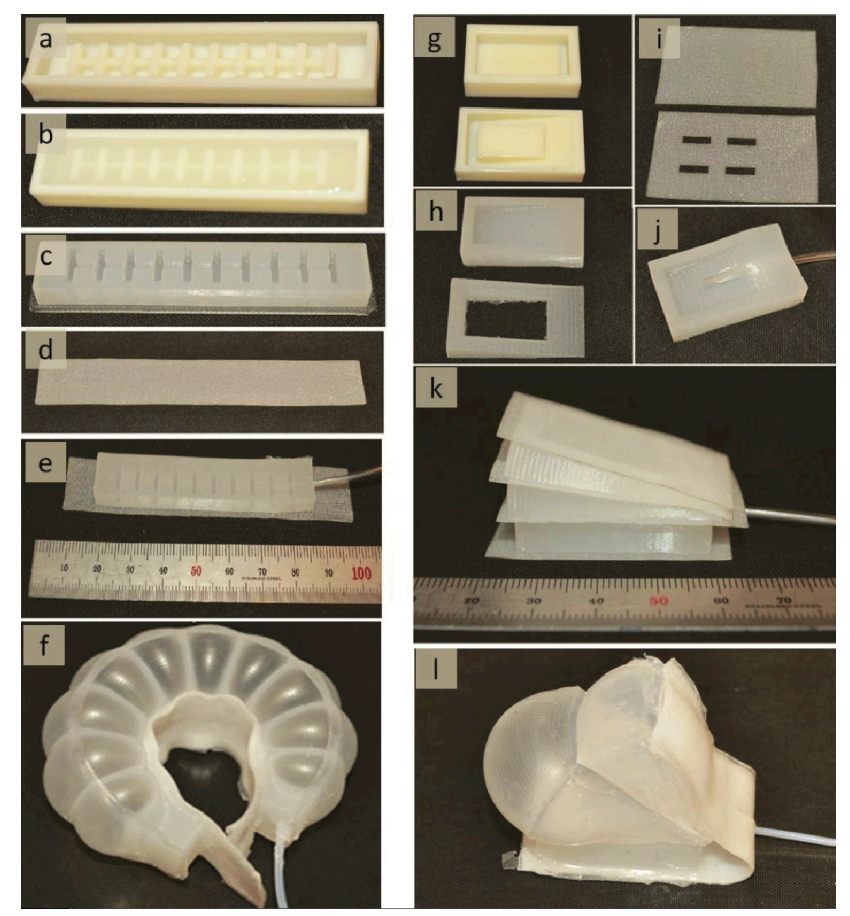

Figure 1 Various SPA designs with different mouldings [17].

\subsection{Smart material-Based Actuators}

There are continuing efforts in using smart materials to create functional actuators for soft robots: shape memory alloy (SMA), shape memory polymer (SMP), ionic polymer metallic composite (IPMC), magneto-rheological fluid, and dielectric elastomers are few of most studied smart materials. Using SMA and SMP as robot's body material has enabled researchers to achieve complicated motions and 
shape transformations in micro-, meso- and even macro-scale robots. Shape memory materials show different behaviour when their environmental condition changes (for most, thermally active stress-strain behaviour is of the most interest for their significant mechanical property change). When SMA passes its phase transition temperature, its phase changes from soft martensite to hard austenite crystal structure. Also while plastic deformation in martensite form is readily possible due to the material atomic structure, in austenite phase, the atomic structure pushes the bulk form of the metal back to its annealed shape. It is claimed that the secondary shape "memory" can be programmed but for practical meso-scale actuation [20], we focus mostly on a single "memory" shape. SMP shows the same behaviour and the difference is that it is softer above its glass transition than in its cold state, and that for meso-scale usage, the strain effect is still minimal relative to SMAs. The use of SMA wires and coils for making soft robots mimicking the peristaltic motion of a worm [21] or an octopus arm [22] have attained much attention already. Another application for these materials is using the elastic behaviour transformation for making adaptive body structures for robots. An example of efforts toward using these materials is a meso-scale wing structure with controllable stiffness joint that can tune its dynamic respond on demand [23]. Other methods of tuning stiffness such as exploiting phase transition in wax coated polymers [24] and phase transition in low melting point metals [25] has also been suggested. Each of these methods has their merits but this variety shows the need for an adjustable stiffness structure that can actively tune the joint stiffness, or turn on / off the existing joint's DoF.

\section{Soft Sensors}

The ultimate sensors for soft robots are thin, stretchable and robust to reconfiguration of the moving body shapes and tasks. Due to the practical cost of adding sensors, robotic manipulators often lack thorough sensor integration and resort to a multi-axis $\mathrm{F} / \mathrm{T}$ sensors at the end effector only. However, a truly conforming, safe and interactive soft robot would require sensors that are soft (stretchable), robust and small enough to be embedded, distributed directly on the robot body. The physical limitations of the sensor material contribute majorly to the difficulties in the fabrication and embed-ability of bendable sensors, let alone stretchable sensors. To overcome this challenge, some of the latest methods are: optimizing hard materials' geometry (aspect ratio, patterns, slits), experimenting with different conductive material, and embedding discrete sensing elements within soft matrix. 


\subsection{Soft Geometry for "Hard" Conductor}

Simply put, strain gauges are a standard example of sensors that have an elevated compliancy and resistive sensitivity due to its planar geometry: dense serpentine shape allows the metal layer to remain flexible. By introducing various types of serpentine patterns, the metal layer can have different sensitivities toward localized curvatures, linear strain, and pressure via resistance change in its effective conductivity. When optimizing geometric patterns, it is not limited to planar surface but also in 3D (the serpentine pattern can be pre-strained to come out of the plane [26]). Stretchable metallization prepares electrodes and conductive tracks; the gold film covered with PDMS shown in [27] stretches up to $20 \%$ of its initial size and measures a pressure up to $160 \mathrm{kPa}$.

\subsection{Conductive material}

A practical way of using conductive material for soft sensors is to make an effective conductive path with a cross-section that would be sensitive to different mechanical loading. There are sensors made of an elastomer with embedded micro channels filled with conductive liquid [28-30]. Upon loading, the electric resistance changes with the deformation of the cross-section areas of the micro channels. Multi-axis strain, bending curvature, normal forces and in-plane (shear forces) can be measured as well $[29,30]$. Carbon nanotube composite thin films have been used as the active sensing material in [31] and these sensors can be stretched up to $2.5 \%$. Conductive polymer based sensors have better sensitivity than the metallic foils while being more flexible. The gauge factor (GF: relative resistance change to the mechanical strain and indicates the sensitivity) of these sensors is 50-100 and 2-5 for the metallic foil sensors. Commercial sensors such as Flexiforce ${ }^{\circledR}$, Bend Sensor ${ }^{\circledR}$, Bi-Flex Sensor ${ }^{\mathrm{TM}}$ also use conductive polymers. Crystalline silicone has been used in [32] which has an active area of $410 \times 410$ $\mu \mathrm{m}^{2}$. The overall size of the sensor is $63 \times 63 \mathrm{~mm}^{2} \times 50 \mu \mathrm{m}$ (thick) with GF of 8.5. Piezo-resistive sensors that use intrinsic piezoelectric effect of the polymer provide the resistance change when subjected to deformations. PeDOT(poly (3,4ethylenedioxythiophene) and conductive ink have been used in [33] which has a GF of 2.48. A micro-structured silicon with DuPont Kapton is used in [34] with a large GF of 43 .

\subsection{Discrete Sensors in Soft Matrix for Distributed Sensing}

When the softness of a sensor is limited by the material property, and if the component size is relatively small, the overall softness can be augmented by 
embedding sensing receptor in a softer matrix. Once the hard "pixel" receptors are embedded in the soft silicone matrix, upon mechanical loading, the receptors provide an electrical output. For this application, piezoelectric materials are desirable for their high sensitivity $(15-65 \mathrm{~nm} / \mathrm{V})$ and consistency under mechanical loading. They are micro-machinable for easy distribution in different size and shape of arrays. Also compared to conventional strain gauges, signal conditioning is easier especially in applications where there are low strains and high noise levels [35]. These are attractive characteristics for applications in wearable and embeddable structures where precise measurement with robustness is required. However, Young's modulus of piezoelectric material is high (2.5 - 63 GPa) and they are very brittle: conditions not ideal for relatively small loading. What we found, however, after embedment in the silicone membrane, the sensitivity is increased due to the large shear loading on the receptor surface [36, 37].

Polysilicon piezoresistive materials are used as strain gauges in many MEMS devices to measure the deflection of a micro-machined deformable structure [3840]: although these materials are robust, have low cost of fabrication, and have a higher gauge factor compared to metal alloy strain gauges, they show nonlinearity with hysteresis. Piezoelectric ceramics (PZT) and piezoelectric polymers such as polyvinylidene fluoride (PVDF) [41-44] are mostly used materials for pressure / force measurements with the piezoelectric effect. To compare PZT and PVDF as sensing receptors, PZTs are less expensive, easier to fabricate, have a high dielectric constant, and provide better electromechanical transformation. However, they are highly brittle. PVDFs are very flexible but have a higher cost of fabrication (easier with $\mathrm{nm}$ wavelength UV laser), lower dielectric constants, lower electromechanical transformation and more signal conditioning for their voltage outputs to be used as a sensor. These sensors are supposed to provide sensing in large areas with distributed arrays. The minimum distance between each piezo receptor depends on the stress distribution are shown in Fig.3 while Fig.4 displays how commercial flexible sensors would look when embedded in the silicone matrix (with PZT receptors, the measuring pixel size can go down to $1.5 \mathrm{x}$ $1.5 \mathrm{~mm}^{2}$ ). The matrix material property determines the stress distribution and dictates overall sensitivity and the resolution of the sensing surface. 


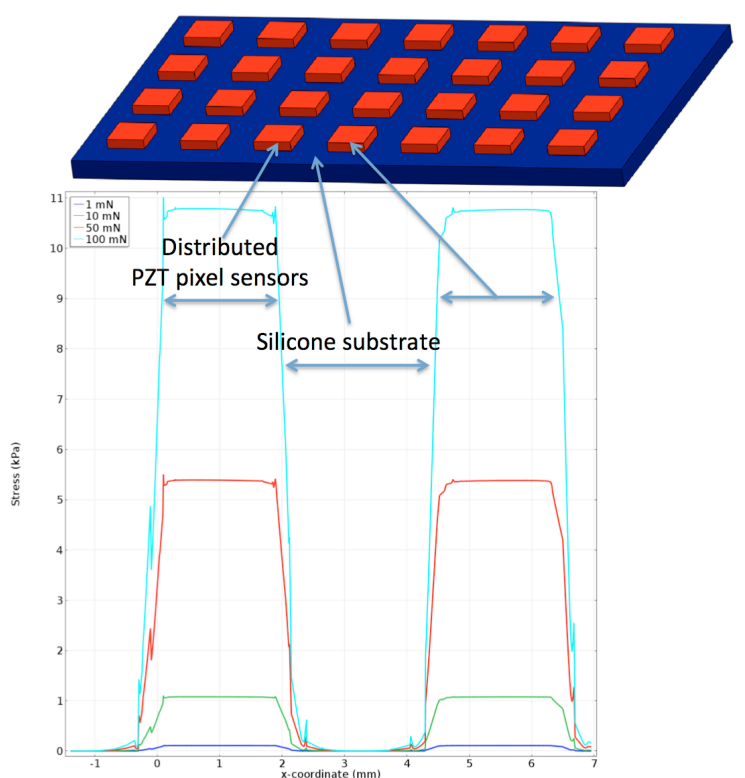

Figure 2 The Sensible Skin with distributed piezoelectric elements embedded in a soft silicone matrix. The graph shows the modulation of stress depending on the input force between the PZT pixels [37].

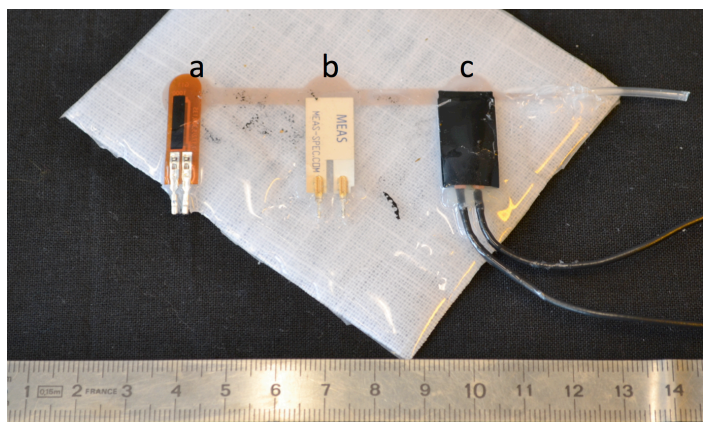

Figure 3 Bending sensors embedded in $500 \mu \mathrm{m}$ thick silicone matrix: a. Flexpoint $^{T M}$, b. piezo film, c. Bi-flex sensor ${ }^{T M}$ [18].

\section{Conclusions}

The foremost hardware challenge of the soft robotics is exploring new solutions toward improving machine-human interaction: this can further be described as task and environmental compliancy, and safety. The need for the new components for soft robots prevail as the community still seeks for the optimal solution toward actuation, sensing, and control as well as total integration. This manuscript 
describes the concurrent research efforts on soft actuation and sensing with promising results, but the remaining challenges are still vast. Now, the investigation of the material and mechanical design from the conception of the robot is the key importance. The application specific functional requirements, the fabrication process of each component must be revisited and refined: this process is particularly important for investigating novel control methods and computational techniques. The design and simulation tools will greatly contribute toward improving the design-fabrication-integration iterations that are undoubtedly labour and experimentally intensive. The technology and research development toward the mentioned soft components will not only advance the robotics community but also neuroprosthetics, materials engineering, chemical engineering, computer sciences and medical studies.

Acknowledgments This work was supported by Swiss NCCR Robotics.

\section{References}

[1] Odhner, Lael U., et al. "A compliant, underactuated hand for robust manipulation." The International Journal of Robotics Research 33.5 (2014): 736-752.

[2] Potratz, Jason, et al. "A light weight compliant hand mechanism with high degrees of freedom." Journal of biomechanical engineering 127.6 (2005): 934-945.

[3] Pratt, Gill A., and Matthew M. Williamson. "Series elastic actuators." Intelligent Robots and Systems 95.'Human Robot Interaction and Cooperative Robots', Proceedings. 1995 IEEE/RSJ International Conference on. Vol. 1. IEEE, 1995.

[4] Tsagarakis, N. G., et al. "A compact soft actuator unit for small scale human friendly robots." Robotics and Automation (ICRA), 2010 IEEE International Conference on. IEEE, 2010

[5] Sensinger, Jonathon W., and Richard Ff Weir. "Improvements to series elastic actuators." Mechatronic and Embedded Systems and Applications, Proceedings of the 2nd IEEE/ASME International Conference on. IEEE, 2006.

[6] Cheng, Nadia, et al. "Design and analysis of a soft mobile robot composed of multiple thermally activated joints driven by a single actuator." Robotics and Automation (ICRA), 2010 IEEE International Conference on. IEEE, 2010.

[7] Onal, Cagdas D., Robert J. Wood, and Daniela Rus. "An origami-inspired approach to worm robots." Mechatronics, IEEE/ASME Transactions on 18.2 (2013): 430-438.

[8] Lee, Dae-Young, et al. "Deformable wheel robot based on origami structure." Robotics and Automation (ICRA), 2013 IEEE International Conference on. IEEE, 2013.

[9] Paik, Jamie K., and Robert J. Wood. "A bidirectional shape memory alloy folding actuator." Smart Materials and Structures 21.6 (2012): 065013.

[10] Hawkes, E., et al. "Programmable matter by folding." Proceedings of the National Academy of Sciences of the United States of America 107.28 (2010): 12441-12445.

[11] Okuzaki, H., et al. "A Biomorphic Origami Actuator Fabricated by Folding a Conducting Paper." Journal of Physics: Conference Series. Vol. 127. 2008.

[12] Daerden, Frank, and Dirk Lefeber. "Pneumatic artificial muscles: actuators for robotics and automation." European journal of mechanical and environmental engineering 47.1 (2002): 11-21.

[13] Beck, Roland D. "Pneumatic actuator control system." U.S. Patent No. 3,237,529. 1 Mar. 1966. 
[14] Tomori, Hiroki, and Taro Nakamura. "Theoretical Comparison of McKibben-Type Artificial Muscle and Novel Straight-Fiber-Type Artificial Muscle." Int. J. Autom. Technol 5.4 (2011): 544-550.

[15] Nakamura, Taro, and Hitomi Shinohara. "Position and force control based on mathematical models of pneumatic artificial muscles reinforced by straight glass fibers." Robotics and Automation, 2007 IEEE International Conference on. IEEE, 2007.

[16] Faudzi, A. M., Razif, M. R. M., Nordin, I. N. A. M., Suzumori, K., Wakimoto, S., \& Hirooka, D. (2012, July). Development of bending soft actuator with different braided angles. In Advanced Intelligent Mechatronics (AIM), 2012 IEEE/ASME International Conference on (pp. 1093-1098). IEEE.

[17] Sun, Yi, Yun Seong Song, and Jamie Paik. "Characterization of silicone rubber based soft pneumatic actuators." Intelligent Robots and Systems (IROS), 2013 IEEE/RSJ International Conference on. Ieee, 2013.

[18] Suh, Chansu and Jamie Paik, "Soft Pneumatic Actuator Skin with Embedded Sensors." Intelligent Robots and Systems (IROS), 2014 IEEE/RSJ International Conference on. Ieee, 2014.

[19] Onal, Cagdas D., and Daniela Rus. "A modular approach to soft robots." Biomedical Robotics and Biomechatronics (BioRob), 2012 4th IEEE RAS \& EMBS International Conference on. IEEE, 2012.

[20] Kohl, Manfred. Shape memory microactuators. Springer, 2004.

[21] Seok, Sangok, et al. "Meshworm: a peristaltic soft robot with antagonistic nickel titanium coil actuators." Mechatronics, IEEE/ASME Transactions on 18.5 (2013): 1485-1497.

[22] Mazzolai, B., et al. "Soft-robotic arm inspired by the octopus: II. From artificial requirements to innovative technological solutions." Bioinspiration \& biomimetics 7.2 (2012): 025005.

[23] Hines, Lindsey, Veaceslav Arabagi, and Metin Sitti. "Shape memory polymer-based flexure stiffness control in a miniature flapping-wing robot." Robotics, IEEE Transactions on 28.4 (2012): 987-990.

[24] Chenel, Thomas, Jamie Paik and Rebecca Kramer. "Variable Stiffness Fabrics with Embedded Shape Memory Materials for Active Joint Stability Braces." Intelligent Robots and Systems (IROS), 2014 IEEE/RSJ International Conference on. Ieee, 2014.

[25] Schubert, Bryan E., and Dario Floreano. "Variable stiffness material based on rigid low-melting-point-alloy microstructures embedded in soft poly (dimethylsiloxane)(PDMS)." Rsc Advances 3.46 (2013): 24671-24679.

[26] Rogers, John A., Takao Someya, and Yonggang Huang. "Materials and mechanics for stretchable electronics." Science 327.5973 (2010): 1603-1607.

[27] Cotton, Darryl PJ, Ingrid M. Graz, and Stephanie P. Lacour. "A multifunctional capacitive sensor for stretchable electronic skins." Sensors Journal, IEEE 9.12 (2009): 2008-2009.

[28] Vogt, Daniel M., Yong-Lae Park, and Robert J. Wood. "Design and Characterization of a Soft Multi-Axis Force Sensor Using Embedded Microfluidic Channels." (2013): 1-1.

[29] Hammond III, Frank L., et al. "Soft Tactile Sensor Arrays for Force Feedback in Micromanipulation." IEEE SENSORS JOURNAL 14.5 (2014): 1443.

[30] Chossat, Jean-Baptiste, et al. "A Soft Strain Sensor Based on Ionic and Metal Liquids." IEEE SENSORS JOURNAL 13.9 (2013): 3405.

[31] Jung, Soyoun, et al. "Flexible strain sensors based on pentacene-carbon nanotube composite thin films." Nanotechnology, 2007. IEEE-NANO 2007. 7th IEEE Conference on. IEEE, 2007.

[32] Zhou, Lisong, et al. "Flexible substrate micro-crystalline silicon and gated amorphous silicon strain sensors." Electron Devices, IEEE Transactions on 53.2 (2006): 380-385.

[33] Correia, Vítor, et al. "Development of inkjet printed strain sensors." Smart Materials and Structures 22.10 (2013): 105028. 
[34] Won, Sang Min, et al. "Piezoresistive strain sensors and multiplexed arrays using assemblies of single-crystalline silicon nanoribbons on plastic substrates." Electron Devices, IEEE Transactions on 58.11 (2011): 4074-4078.

[35] Sirohi, Jayant, and Inderjit Chopra. "Fundamental understanding of piezoelectric strain sensors." Journal of Intelligent Material Systems and Structures 11.4 (2000): 246-257.

[36] Roche, Denis, et al. "A piezoelectric sensor performing shear stress measurement in an hydrodynamic flow." Applications of Ferroelectrics, 1996. ISAF'96., Proceedings of the Tenth IEEE International Symposium on. Vol. 1. IEEE, 1996.

[37] Acer, M, Marco Salerno and Jamie Paik, "Piezo resistive sensors with Silicone Embedment." Submitted for publication.

[38] H, Yating, Katragadda, Rakesh B, TU, Hongen, et al. "Bioinspired 3-D tactile sensor for minimally invasive surgery.” Microelectromechanical Systems, Journal of, 2010, vol. 19 , no 6 , p. $1400-1408$.

[39] Ahmed, M. Chitteboyina, M.M., Butler, D.P. and Celik-Butler, Z., "MEMS Force Sensor in a Flexible Substrate Using Nichrome Piezoresistors," Sensors Journal, IEEE, vol.13, no.10, pp.4081,4089, Oct. 2013

[40] Noda, K., Hoshino, K., Matsumoto, K., \& Shimoyama, I. (2006). “A shear stress sensor for tactile sensing with the piezoresistive cantilever standing in elastic material". Sensors and Actuators A: physical, 127(2), 295-301.

[41] Ottermo, Maria V., Oyvind Stavdahl, and Tor Arne Johansen. "Palpation instrument for augmented minimally invasive surgery." Intelligent Robots and Systems, 2004. (IROS 2004). Proceedings. 2004 IEEE/RSJ International Conference on. Vol. 4. IEEE, 2004.

[42] Seminara, Lucia, et al. "Piezoelectric Polymer Transducer Arrays for Flexible Tactile Sensors." IEEE Sensors Journal 13.10 (2013).

[43] Qasaimeh, M. A., S. Dargahi, and M. J Kahrizi. "pvdf-based microfabricated tactile sensor for minimally invasive surgery." Microelectromechanical Systems, Journal of (2009).

[44] Zirkla, M., et al. "PyzoFlex®: a printed piezoelectric pressure sensing foil for human machine interfaces." Proc. of SPIE Vol. Vol. 8831. 2013. 